\title{
Protección pulpar: cementos a base de silicato de calcio. Relato de un caso clínico
}

\author{
Pulp capping: calcium silicate-based cements. Report of a clinical case \\ Joshua Anggelo Yfuma Pedroza1,a, Romina Andrea Naupari Villasante 1,b, Andres Kenichi Noborikawa \\ Kohatsu ${ }^{1, c}$, Miguel Manuel Rolando Nuñez Gamboa d, 2,e;,;
}

\section{RESUMEN}

Los cementos a base de silicato de calcio son materiales bioactivos, que poseen la capacidad de liberar mayor cantidad de iones de calcio e hidroxilo, brindando ciertas ventajas como mayor capacidad antimicrobiana y de remineralización, incrementando la longevidad de los tratamientos pulpares. Theracal LC es un cemento a base de silicato de calcio el cual tiene como ventaja su presentación de fácil aplicación a comparación de otros cementos bioactivos. En el presente reporte de caso, Theracal LC fue utilizado como forro cavitario. Al examen clínico el paciente presentó las piezas 36 y 35 con el diagnóstico dental: caries dental con lesión con cavidad y caries dental con lesión profunda adyacente a restauración, respectivamente y como diagnóstico pulpar: Pulpa sana, en ambas piezas. Se realizaron restauraciones directas con resina compuesta en las piezas 36 y 35 , considerando los siguientes pasos: grabado selectivo, sistema adhesivo autocondicionante, cemento protector a base de silicato de calcio y resina compuesta para técnica Bulk.

PALABRAS CLAVE: recubrimiento de la pulpa dental, cemento de silicato, hidróxido de calcio.

\section{SUMMARY}

Calcium silicate-based cements are bioactive materials, which have the capacity to release a greater number of calcium and hydroxyl ions, providing some advantages like an increased antimicrobial and remineralise activity, increasing the pulp treatments longevity. Theracal LC is a calcium silicate-based cement that has as an advantage its easy application, comparing to others bioactive cements. In this case report, Theralcal LC was used as a cavity liner. At clinical examination teeth 36 and 35 exhibited diagnosis of Dental caries; 36 with a cavitated lesion and 35 with a lesion adjacent to a previous restoration; and pulp diagnosis, Sound Pulp, for both tooth pieces. Direct restorations were made with composite resin in tooth pieces 36 and 35, considering the following steps: selective etching, self-etch adhesive system, calcium silicate-based cement and Bulk-fill resin composite.

KEYBORDS: Dental pulp capping, silicate cement, calcium hydroxide.

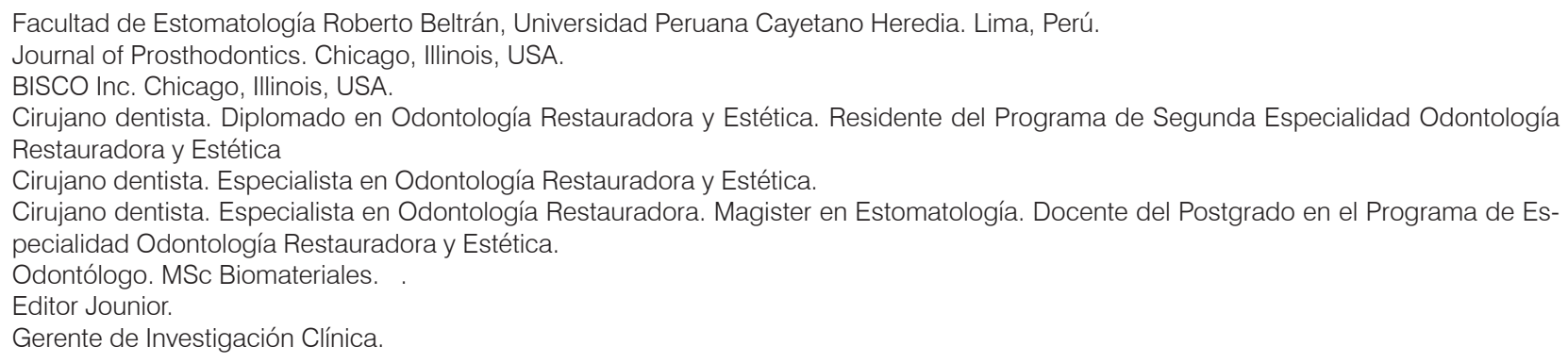




\section{INTRODUCCIÓN}

El tratamiento restaurador tiene como objetivo preservar la vitalidad pulpar, lo cual permite aumentar la resistencia mecánica y supervivencia de la pieza dentaria a largo plazo (1). Para prolongar el tiempo de vida de una pieza dentaria se tiene en cuenta tratamientos conservadores como el recubrimiento pulpar indirecto y directo. Este último procedimiento presenta una tasa de éxito que varía desde $31,8 \%$ - 91,3\% (2), esta tasa de éxito va depender de la evaluación diagnóstica del tejido pulpar y del material de elección.

Actualmente la clasificación clínica de patología pulpar de la Asociación Americana de Endodoncia (AAE) refiere que los términos de Pulpitis Reversible e Irreversible, se diferencian principalmente en la capacidad del tejido pulpar inflamado en retomar su normalidad, clínicamente se diferencian por la ausencia de dolor espontáneo o por la ausencia de dolor después de retirar el estímulo (3).

Los materiales a base de hidróxido de calcio son el estándar de oro de las terapias pulpares porque presentan como ventajas ser antibacteriano y promover la formación de tejido duro. Estos materiales también presentan dos principales desventajas que son la solubilidad en los fluidos tisulares y los defectos de túneles que se producen en la formación de puentes dentinarios (4). Otros materiales como los cementos a base de silicato de calcio se han comercializado con el

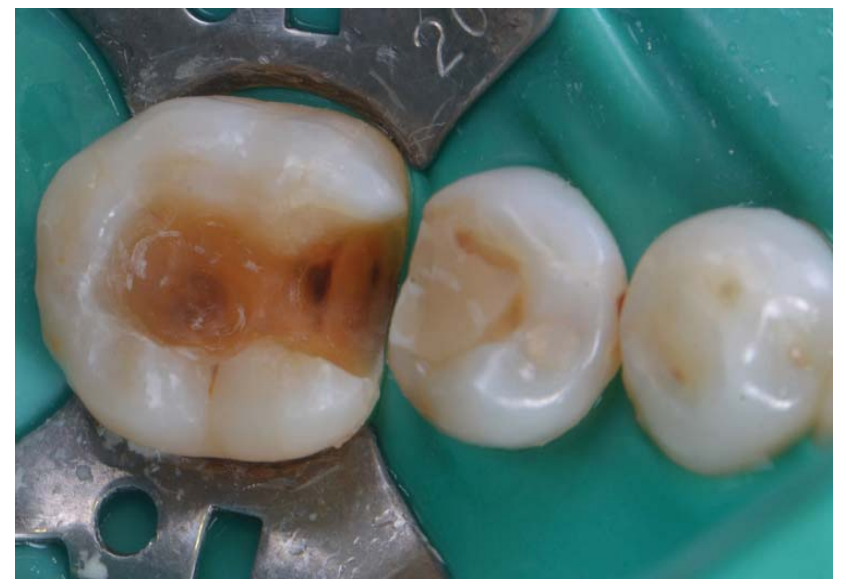

Figura 1. Diseño cavitario para futura restauración. objetivo de mejorar estas desventajas, son materiales bioactivos capaces de formar apatita mediante el uso del silicato de calcio o aluminato de calcio, liberan iones de calcio los cuales estimulan la formación del puente dentinario y han demostrado superioridad con respecto al hidróxido de calcio. Entre estos materiales tenemos al Agregado de Trioxido de Mineral (MTA), Biodentine (Septodont, Saint Maur des Fosses, Francia), Theracal LC (Bisco, Schamburg, IL, EE.UU.) y Theracal PT (Bisco, Schamburg, IL, EE.UU.) (5).

El objetivo del presente caso fue devolver al paciente la función masticatoria y estética teniendo en consideración la vitalidad pulpar del paciente, con el uso de un cemento a base de silicato de calcio como es el Theracal LC.

\section{Reporte de caso}

Paciente de 40 años de edad con buen estado de salud general, sin riesgo al tratamiento estomatológico; al examen clínico intraoral presenta cavidad con lesión cariosa en pieza dental 36 y restauración de resina compuesta en mal estado en pieza dental 35. Se realizó la remoción del tejido dental cariado en la pieza 36 , con previa colocación del aislamiento absoluto, y se dejó la cavidad preparada con ángulos internos redondeados, paredes lisas y expulsivas (figura 1).

Preparación química de la superficie dental: Se realizó grabado ácido selectivo con ácido fosfórico 35\% Select HV (Bisco, Schamburg, IL, EE.UU.) (figura 2),

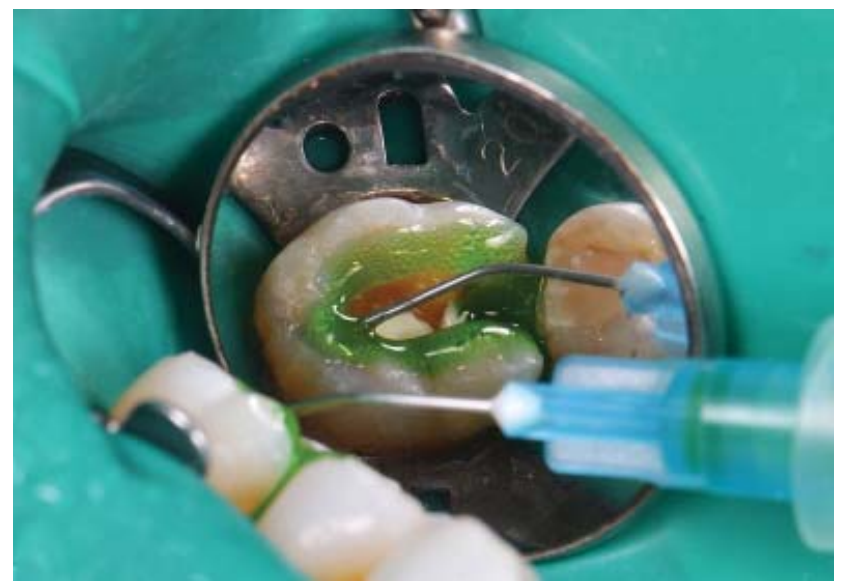

Figura 2. Grabado ácido selectivo 


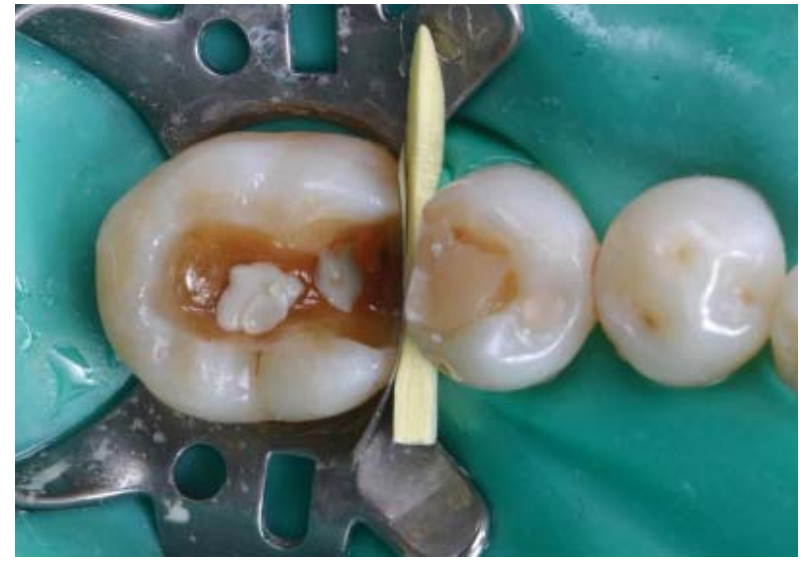

Figura 3. Aplicacion de sistema adhesivo posterior a la aplicación del protector pulpar

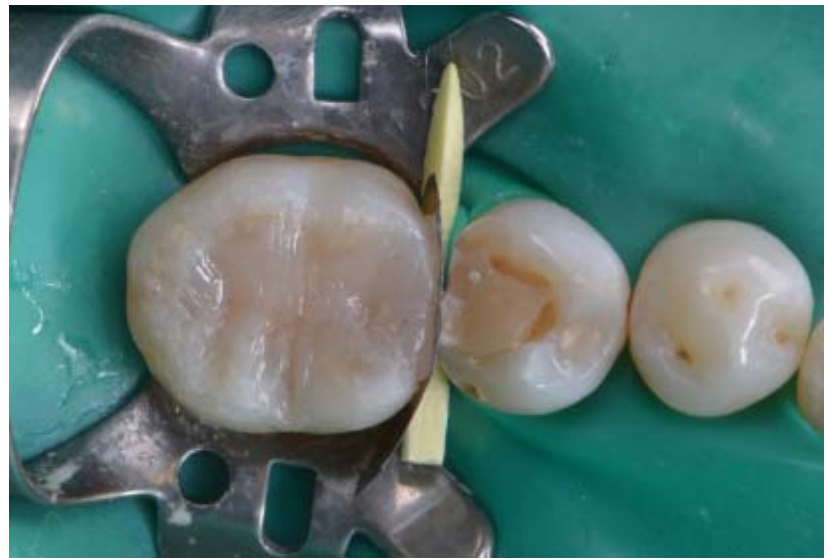

Figura 5. Restauración finalizada

y se aplicó sistema adhesivo autoacondicionante de dos pasos QuickBond (Itena Clinical, Paris, Francia). Previo a la aplicación del sistema adhesivo se colocó una capa de cemento protector pulpar a base silicato de calcio TheraCal LC (Bisco, Schamburg, IL, EE.UU.), principalmente en las zonas más profundas de la cavidad ( $>2-3 \mathrm{~mm}$ de profundidad) como forro protector, siguiendo el protocolo sugerido por el fabricante: dentina húmeda, capas de máximo $1 \mathrm{~mm}$ de grosor y fotopolimerización por 20 seg (figura 3 ).

Restauración de la cavidad: Para la restauración se utilizó resina compuesta para técnica Bulk SonicFill (Kerr,EE.UU.). Se consideró realizar la restauración con técnica centrípeta, para facilitar la técnica restauradora (figura 4). A pesar de poder realizar incrementos grandes se realizaron 8 incrementos incluyendo las cúspides, debido a que la

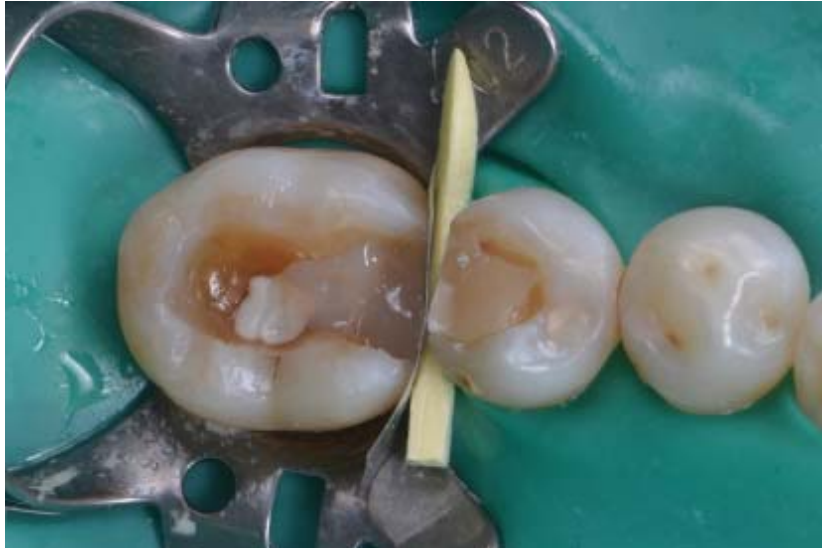

Figura 4. Primer incremento de la pared proximal siguiendo la técnica centripeta

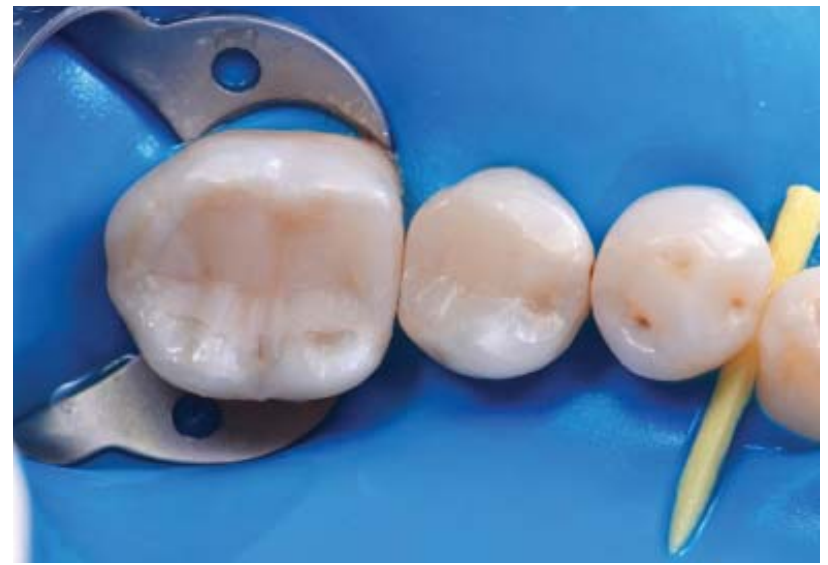

Figura 6. Restauraciones de la pieza 36 y 35 finalizadas con acabado y pulido

polimerización disminuye considerablemente entre las capas más profundas. Los últimos incrementos se realizaron insertándolos en cúspides oblicua y opuestas para disminuir el estrés de contracción del material (figura 5).

Acabado y pulido: Se realizó la eliminación de excesos de resina compuesta con una punta diamantada en forma de fisura de granulación fina, dejando la restauración con una adecuada anatomía y lista para el ajuste oclusal y pulido final de la restauración con el sistema de pulido Diatech Shape Guard (Coltène, Altstatten, AG, Suiza). Finalmente, se procedió a restaurar la pieza dental 35 utilizando los mismos materiales y protocolos restauradores mencionados para la pieza dental 36 (figura 6). 


\section{DISCUSIÓN}

En la actualidad no se ha encontrado un protocolo ideal que los odontólogos restauradores puedan seguir para preservar la vitalidad pulpar. El procedimiento de Recubrimiento Pulpar Indirecto como Recubrimiento Pulpar Directo, son procedimientos en los cuales se aplica un material terapéutico para poder promover la remineralización, mantener la vitalidad y proteger el tejido pulpar de diferentes estímulos nocivos, térmicos y químicos $(6,7)$.

Theracal LC es un material a base de silicato de calcio de cuarta generación modificado con resina, es un cemento de pasta única promovido por su fabricante como recubridor pulpar directo e indirecto. Según la norma ISO 9917-2017, cláusula 4.1 de la parte 2 el Theracal LC es un cemento de clase 2, el cual la reacción de fraguado del componente polimerizable es activado por luz (8).

Gandolfi et al., mencionan que este material tiene la capacidad de liberar iones de calcio que están en forma de hidroxido de calcio; lo cual permite la formación de apatita de calcio. Se ha demostrado que este material presenta una mayor capacidad de liberación de iones de calcio en comparación con el hidróxido de calcio fraguable (pasta-pasta) (9). En estudios in vitro de hasta 28 días, Theracal LC presenta similar capacidad de liberación de calcio y formación de apatita comparado con otros materiales a base de silicato de calcio como el ProRoot MTA (Dentsply, Johnson City, TN, EE.UU.) sin cambios estadísticamente significativos a lo largo del tiempo. Del mismo modo, la cantidad liberada de iones calcio e hidroxilo permite la formación de un adecuado puente dentinario, debido a la estimulación de odontoblastos a nivel del tejido pulpar $(5,8,9)$.

Una ventaja adicional del Theracal LC es su pH alcalino de 10.66, el cual después de 3 horas de su aplicación y después de las 24 horas, se mantiene estable sin cambios estadísticamente significativos en ambos tiempos. Esta alta alcalinidad ambiental permite que el material tenga una buena capacidad antimicrobina como lo presenta el el hidróxido de calcio fraguable (pasta-pasta) o el ProRoot MTA; también una excelente propiedad biológica, que se da por la formación o precipitación de calcio y fosfato en la interfaz del material - dentina produciendo el sellado de la misma $(5,10,11)$.
Otra característica importante es su menor capacidad de solubilidad, la cual contribuye a un mejor sellado biológico mediante enlaces químicos con la dentina, esta unión íntima con la dentina permite que se de la liberación de iones de calcio e hidroxilo para formación de la apatita de calcio. Algunos estudios han demostrado que este material tiene menor solubilidad y mejor capacidad de sellado en comparación con los siguientes materiales: Biodentine, ProRoot MTA, Angelus MTA y el el hidróxido de calcio fraguable (pasta-pasta) $(9,11,12)$.

El Theracal LC, al ser un material que contiene monómeros de acrílico como el BIS-GMA, presenta una biocompatibilidad cuestionable en los tratamientos pulpares directos. La mayoría de estudios han señalado un menor desempeño clínico de este material en comparación con sus homólogos como Retro MTA, ProRoot MTA y Biodentine, cuando es aplicado directamente al tejido pulpar en pulpotomías parciales. Sin embargo, se tiene en consideración la dificultad de ser concluyentes en este aspecto, debido a la influencia de otros factores en el éxito de una terapia pulpar directa como, por ejemplo: la proximidad de la lesión cariosa, la vitalidad pulpar, edad del paciente y la asepsia durante el tratamiento $(13,14,15)$.

El Theracal LC según sus indicaciones se puede utilizar como forro cavitario y como protector pulpar directo e indirecto. Como hay evidencia científica de que los monómeros pueden ser citotóxicos cuando entran en contacto con el tejido pulpar, su uso más frecuente es como forro cavitario y protector pulpar indirecto. Últimamente se ha introducido el Theracal PT, que es un material muy silmilar al Theracal LC, la diferencia entre ellos radica en su método de curado, ya que su mecanismo de curado es dual permitiendo un curado más completo en 5 minutos, en zonas en las cuales la energía de la luz no llega por completo, esta ventaja permite ser indicado en el recubrimiento pulpar directo; del mismo modo presenta una formulación patentada lo cual permite ser un material de fotocurado como el Theracal LC y a la vez mantener la facilidad en su aplicación debido a su excelentes propiedades tixotrópicas. Aunque aún no existen estudios disponibles que respalden su uso, este material esta indicado principalmente para pulpotomías, tambien está indicado en recubrimiento pulpar directo e indirecto según el fabricante. Como odontólogos no solo debemos estar informados sobre los materiales 
existentes sino también estar informados sobre los nuevos materiales, sus indicaciones y sus posibles resultados prometedores (16).

\section{CONCLUSIONES}

Los protectores pulpares a base de silicato de calcio han demostrado superioridad a comparación del hidróxido de calico, tienen mayor capacidad de liberación de iones de calcio e hidroxilos, lo que permite una óptima formación del puente dentinario. Entre los materiales a base de silicato de calcio, el TheraCal LC ha demostrado resultados muy similares a los presentados por el Biodentine y MTA, este material es de fácil aplicación porque viene en un dispensador directo y no se tiene que realizar algún tipo de mezcla previa o la necesidad de un aparato para mezclar como el amalgamador.

El único resultado cuestionable es con respecto a su citotoxicidad debido a la inclusión de monómeros de resina en su composición, por lo cual su aplicación como recubridor pulpar directo es un tema aún no definido, hasta que existan más estudios in vitro e in vivo que lo respalde.

\section{REFERENCIAS BIBLIOGRAFICAS}

1. Yu C, Abbott PV. An overview of the dental pulp: its functions and responses to injury. Aust Dent J. 2007;52: 4-16.

2. Bjørndal L, Reit C, Bruun G, et al. Treatment of deep caries lesions in adults: randomized clinical trials comparing stepwise vs direct complete excavation, and direct pulp capping vs partial pulpotomy. Eur J Oral Sci. 2010;118:290-7.

3. Glickman GN. AAE Consensus Conference on Diagnostic Terminology: background and perspective. J Endod. 2009;35(12):1619-20.

4. Kurun M, Tulga F, Orhan K. Evaluation of calcium $(\mathrm{Ca} 2+)$ and hydroxide $(\mathrm{OH}-)$ ion diffusion rates of indirect pulp capping materials. Int J Artif Organs. 2017;40(11):641-646.

5. Dawood AE, Parashos P, Wong RHK, Reynolds EC, Manton DJ. Calcium silicate-based cements: composition, properties, and clinical applications. Investig Clin Dent. 2017;8(2): 1-15.

6. Hilton TJ. Keys to clinical success with pulp capping: a review of the literature. OperDent. 2009;34(5):615-25.

7. Arandi NZ. Calcium hydroxide liners: a literature review. Clin Cosmet Investig Dent. 2017;9:67-72.

8. Arandi NZ, Rabi T. TheraCal LC: From Biochemical and Bioactive Properties to Clinical
Applications. Int J Dent. 2018; 2018:3484653.

9. Gandolfi MG, Siboni F, Botero T, Bossù M, Riccitiello F, Prati C. Calcium silicate and calcium hydroxide materials for pulp capping: biointeractivity, porosity, solubility and bioactivity of current formulations. J Appl Biomater Funct Mater. 2015;13(1):43-60.

10. Poggio C, Lombardini M, Colombo M, Beltrami $\mathrm{R}$, Rindi S. Solubility and $\mathrm{pH}$ of direct pulp capping materials: a comparative study. J Appl Biomater Funct Mater. 2015;13(2):e181-5

11. Yamamoto S, Han L, Noiri Y, Okiji T. Evaluation of the $\mathrm{Ca}$ ion release, $\mathrm{pH}$ and surface apatite formation of a prototype tricalcium silicate cement. Int Endod J. 2017;50 (Suppl 2):e73-e82.

12. Makker S, Kaur H, Aggarwal A, Vashisht R. AConfocal laser scaninng microscopic study evaluating the sealing ability of mineral trioxide aggregate, biodentine and a new pulp capping agent - Theracal. Dental Journal of Advace Studies. 2015; 20(25):1482-2321.

13. Lee H, Shin Y, Kim SO, Lee HS, Choi HJ, Song JS. Comparative study of pulpal responses to pulpotomy with proroot MTA, RetroMTA, and TheraCal in Dogs' Teeth. J Endod. 2015;41(8):1317-24.

14. Jeanneau C, Laurent P, Rombouts C, Giraud T, About I. light-cured tricalcium silicate toxicity to the dental pulp. J Endod. 2017;43(12):2074-2080.

15. Bakhtiar $\mathrm{H}, \mathrm{Nekoofar} \mathrm{MH}, \mathrm{Aminishakib} P$, et al. Human pulp responses to partial pulpotomy treatment with TheraCal as compared with biodentine and ProRoot MTA:Aclinicaltrial.JEndod.2017;43(11):1786-1791.

16. Bisco Inc. TheraCal PTт: Dual-Cured ResinModified Calcium Silicate Pulpotomy Treatment. Illinois: BISCO; 2019. (Fecha de acceso: 19 de noviembre del 2019) Disponible en: https://www. bisco.com/assets/1/22/TheraCal_PT_English.pdf

Recibido: 21-01-20

Aceptado: 19-06-20 